\title{
Frequent vomiting attacks in a patient with Lhermitte-Duclos disease: a rare pathophysiology of cerebellar lesions?
}

\author{
Chika Somagawa, MD, ${ }^{1}$ Tomonori Ono, MD, PhD, ${ }^{2,3}$ Ryoko Honda, MD, ${ }^{3,4}$ Hiroshi Baba, MD, PhD, ${ }^{5}$ \\ Takeshi Hiu, MD, PhD, ${ }^{2}$ Ryujiro Ushijima, MD, ${ }^{2}$ Keisuke Toda, MD, PhD, ${ }^{2,3}$ Kei Sato, MD, ${ }^{2}$ \\ Masahiro Ito, MD, PhD, ${ }^{6}$ and Keisuke Tsutsumi, MD, PhD ${ }^{2}$
}

\begin{abstract}
${ }^{1}$ Residency Program, ${ }^{2}$ Department of Neurosurgery, ${ }^{3}$ Epilepsy Center, and ${ }^{4}$ Department of Pediatrics, National Nagasaki Medical Center, Omura; ${ }^{5}$ Nishi-Isahaya Hospital, Isahaya; and ${ }^{6}$ Department of Pathology, National Nagasaki Medical Center, Omura, Nagasaki, Japan
\end{abstract}

\begin{abstract}
Lhermitte-Duclos disease (LDD) is a neurological disease caused by a hamartomatous lesion in the cerebellum. Clinically, LDD is commonly associated with progressive space-occupying lesion effects in the posterior fossa, increasing intracranial pressure, occlusive hydrocephalus, and focal neurological deficits of adjacent structures. The authors report the case of a 10-year-old boy with LDD who had been suffering from vomiting attacks (VAs). These VAs had been brief in duration but extremely frequent, and they had been resistant to antiemetic drugs since the early postnatal period. Magnetic resonance imaging at 8 months of age revealed a right cerebellar lesion with very little space-occupying lesion effect, but the causal relationship with VAs was not evident at that point, because no clinical symptoms or signs other than vomiting were suggestive of increased intracranial pressure. The VAs were initially diagnosed as autonomic ataxia and had been treated with antiemetic drugs for approximately 10 years, but the patient's symptoms were not improved at all in frequency or duration. He developed convulsive seizures at 9 years of age and was referred to the authors' epilepsy center. The VAs were initially speculated to represent an aspect of seizures, but antiepileptic agents proved ineffective against this symptom despite remission of convulsive seizures. Video-electroencephalography monitoring did not show any evolving ictal patterns associated with the vomiting. Careful reevaluation of MRI studies revealed that the cerebellar lesion was fused with the cerebellum, middle and inferior cerebellar peduncles, and dorsolateral medulla oblongata with some distortion. FDG-PET identified hypermetabolism in the cerebellar lesion. After establishing the diagnosis of LDD, the authors performed subtotal resection of the lesion based on the likelihood of a causal relationship between the cerebellar lesion and the vomiting center of the medulla oblongata. Postoperatively and for 2 years, VAs have remained completely suppressed. The authors hypothesize that the pathophysiology of VAs in LDD includes a tumor-like spaceoccupying effect on the vomiting center of the medulla oblongata, and even partial resection of the lesion may prove effective.
\end{abstract}

https://thejns.org/doi/abs/10.3171/2017.4.PEDS1735

KEY WORDS Lhermitte-Duclos disease; vomiting; medulla oblongata; surgical treatment; pathophysiology; cerebellum; congenital

$\mathrm{L}$ HERMITTE-Duclos disease (LDD) is a neurological disease caused by a hamartomatous lesion in the cerebellum. ${ }^{9}$ Clinical occurrence is most frequent among young adults, but individuals of all ages can be affected. ${ }^{9}$ LDD is commonly associated with a progressive space-occupying lesion (SOL) effect in the posterior fossa, as with other cerebellar mass lesions, typically causing increased intracranial pressure (ICP), occlusive hydro- cephalus, and focal neurological deficits of adjacent structures. ${ }^{9}$ Cerebellar dysfunctions such as ataxia, gait disturbance, nystagmus, and nausea/vomiting are occasionally observed. ${ }^{1,9}$ However, no reports have described frequent vomiting attacks (VAs) accompanying LDD that continued from birth and that were not attributable to increased ICP. We describe the case of a 10-year-old boy with LDD who had been suffering from frequent VAs since the early

ABBREVIATIONS ICP = intracranial pressure; $L D D=$ Lhermitte-Duclos disease SOL = space-occupying lesion; $V A=$ vomiting attack.

SUBMITTED January 21, 2017. ACCEPTED April 10, 2017.

INCLUDE WHEN CITING Published online June 23, 2017; DOI: 10.3171/2017.4.PEDS1735. 

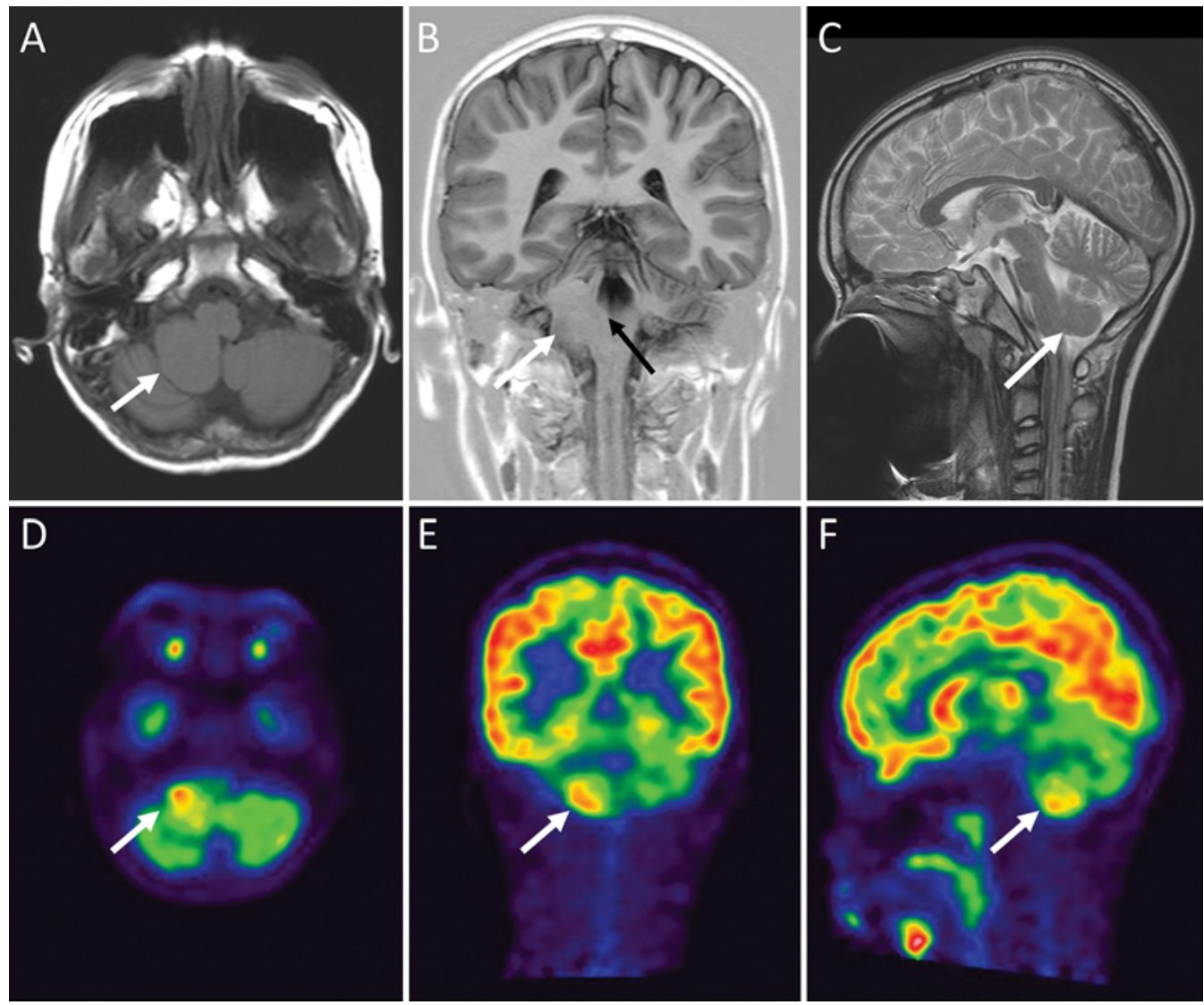

FIG. 1. Preoperative MRI and ictal FDG-PET studies. Axial T1-weighted (A), coronal T1-weighted inversion recovery (B), and sagittal T2-weighted (C) MR images showing the right cerebellar lesion (white arrows) fused to the pons and medulla oblongata involving the right middle and inferior cerebellar peduncle and the lower limit of the fourth ventricle at the area postrema (black arrow). Axial (D), coronal (E), and sagittal (F) FDG-PET scans obtained during VAs and corresponding to each MRI plane, showing hypermetabolic activity localized to the lower part of the cerebellar lesion (white arrows). Figure is available in color online only.

neonatal period and who was successfully treated with partial lesionectomy, and we discuss the pathophysiology involved in the case.

\section{Case Report}

\section{History}

The patient was a developmentally appropriate 10 -yearold boy. He had been born through vaginal delivery at a gestational age of 39 weeks and had a history of complex febrile seizures starting at 2 years of age. Unprovoked VAs occurred frequently in the early neonatal period. At that time the attacks lasted only a few minutes and occurred almost hourly during wakefulness. Repeated metabolic screening tests and gastrointestinal tract investigations revealed no cause of the VAs. The patient's head circumference and neurodevelopmental course were normal. MRI at 8 months of age identified a lesion thought to be a hamartoma in the right cerebellum, but no causal relationship with the VAs was suspected at that time. The VAs were initially diagnosed as autonomic ataxia and had been treated with antiemetic drugs for approximately 10 years, but the patient's symptoms showed no improvements at all in frequency or duration. Repeated MRI did not show any progressing features.
At 9 years of age, the patient experienced an afebrile generalized tonic-clonic seizure that recurred once a month despite antiepileptic treatment with carbamazepine and valproic acid.

\section{Examination}

At 10 years of age, the patient was referred to our epilepsy center for evaluation of the seizures. He showed mild cerebellar ataxia and dysmetria of the right side of his body in the period between VAs. The VAs appeared suddenly, without any clear initiating causes such as eating, motion, or position of the body, and they lasted only a few minutes. No other episodic cerebellar signs such as ataxia, vertigo, or nystagmus were evident during VAs. Long-term video-electroencephalography monitoringusing a standard 10-20 montage-recorded no epileptic abnormalities during the attacks or intermittent periods. Although generalized tonic-clonic seizures were controlled with zonisamide, the VAs persisted. MRI showed a cerebellar lesion fused to the right middle and inferior cerebellar peduncle without any SOL effect, but with some distortion of the dorsolateral medulla oblongata, as had been shown on MRI studies at the previous hospital. No cerebral lesions or hydrocephalus were revealed (Fig. 

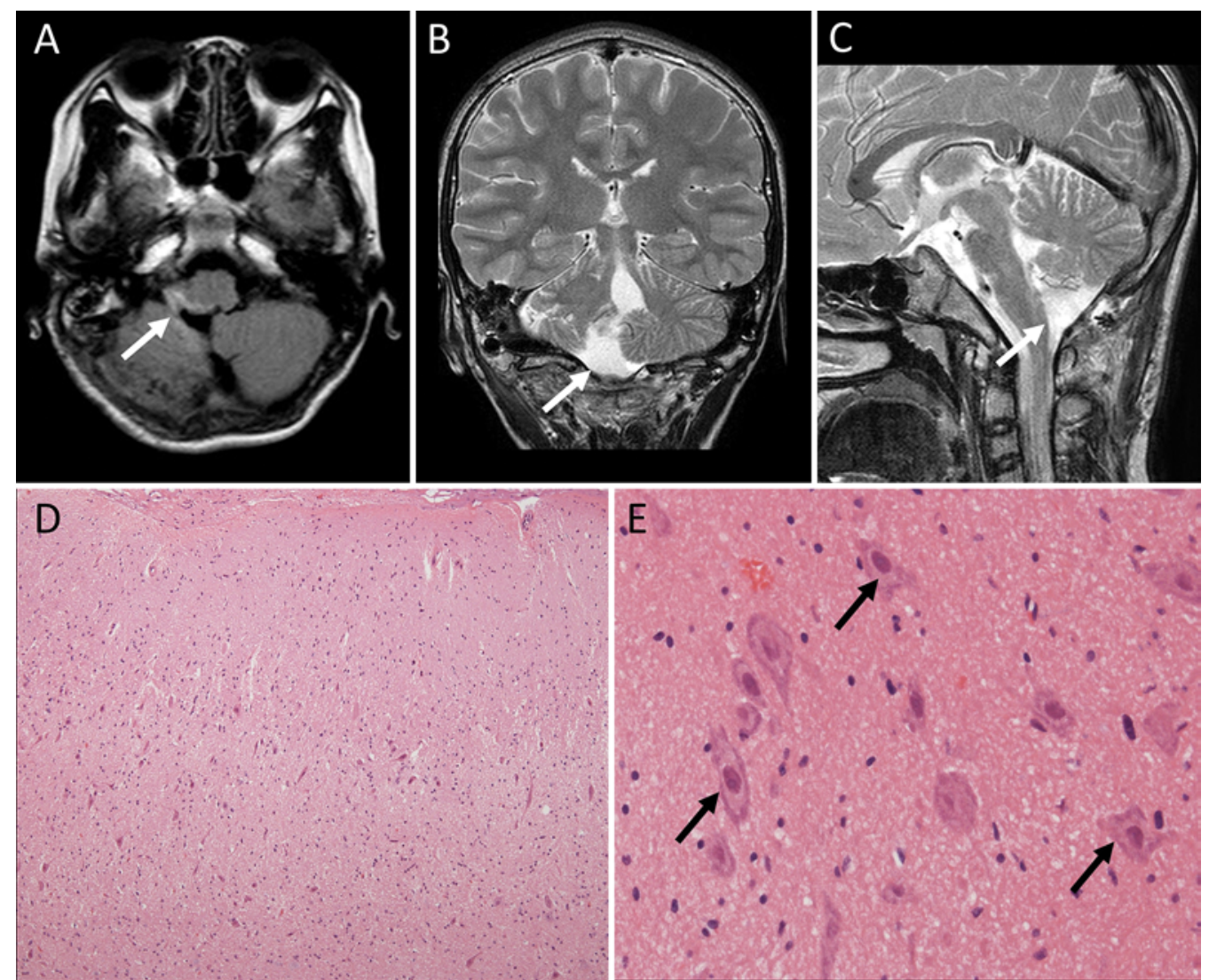

FIG. 2. Postoperative MRI and histopathological features of resected tissue. Axial FLAIR (A), coronal T2-weighted (B), and sagittal T2-weighted (C) MR images showing subtotal resection of the lower part of the cerebellar lesion (white arrows). Low- and high-power ( $\boldsymbol{D}$ and $\mathbf{E}$, respectively) photomicrographs of $\mathrm{H} \& \mathrm{E}$-stained resected tissue revealing the disappearance of the normal cerebellar architecture, including granular and Purkinje cells replaced by large ganglion cells (black arrows). This finding is consistent with dysplastic gangliocytoma of the cerebellum. Original magnification $\times 10(\mathrm{D})$ and $\times 40(\mathrm{E})$. Figure is available in color online only.

1). FDG-PET showed hypermetabolism localized to the lesion (Fig. 1). We diagnosed LDD and speculated that the patient's VAs were caused by the cerebellar lesion seen on MRI and FDG-PET.

\section{Operation}

We performed subtotal lesionectomy via a lateral suboccipital approach when the patient was 11 years old (Fig. 2). Intraoperatively we observed that the lesion was completely fused with the right cerebellum. The surrounding cranial nerves appeared normal and unaffected. To preserve the middle cerebral peduncle, the lower part of the lesion below the level of cranial nerves VII and VIII was resected in piecemeal fashion. The lesion was also fused with the dorsolateral medulla oblongata, with no clear limit. The lower floor of the fourth ventricle and the obex were exposed after resection, resulting in removal of the lower portion of the cerebellar peduncle.

\section{Postoperative Course and Follow-Up}

The VAs disappeared immediately after surgery and no relapse has been seen for more than 2 years postoperatively. Transient aggravation of right cerebellar signs and gait disturbance were observed, but the patient made a full recovery from both within 2 months. Two years after sur- gery, the patient now enjoys daily living and school activities without any disabling symptoms. Histopathological findings were consistent with cerebellar gangliocytoma (Fig. 2).

\section{Discussion}

The clinical presentation in LDD is typically attributed to an SOL effect or increased ICP-i.e., headache, cerebellar ataxia, cranial nerve palsies, disturbance of consciousness, or nausea/vomiting. ${ }^{9}$ In our patient, the VAs were unaccompanied by increased ICP. The pattern of the VAs-i.e., long-term repetition of brief episodes from the early neonatal period-was considered unlikely to have been caused by increased ICP.

Vomiting can also be caused by SOL effects in the cerebellum, is often associated with disequilibrium and vertigo mimicking vestibular dysfunction, and is likely to be triggered by motion and position. ${ }^{6}$ However, the VAs in our patient were independent of those triggers, and neurological examination showed only mild chronic cerebellar signs.

Vomiting is a reflective symptom mediated by the CNS. The vomiting center in the medulla oblongata comprises the lateral reticular formation and nucleus solitarius. ${ }^{2-4}$ It can be stimulated by diverse afferent impulses from the 
gastrointestinal tract and brain, and reflexive efferent impulses to the peripheral organs induce vomiting. ${ }^{2-4,10}$

In our patient MRI demonstrated that the cerebellar lesion appeared to be fused with and to slightly distort the dorsolateral medulla oblongata, involving the right lower limit of the fourth ventricle where the area postrema is located as a chemoreceptor trigger zone. One possible mechanism thus might have involved mechanical stimulation of this structure by the lesion.

Why the symptoms in our patient occurred intermittently and repeated in a seizure-like manner remains unclear. Another hypothesis is neuronal irritation of the vomiting center through network connections. However, direct pathways between the cerebellum and reticular formation in the medulla oblongata have not been found in humans. ${ }^{3}$ Three cases of patients with cerebellar hamartoma or ganglioglioma who experienced hemifacial seizures have been reported..$^{5,7}$ Ictal SPECT demonstrated hyperperfusion at the lesion in each of those 3 patients. In addition, in one patient, repetitive theta rhythmic discharges were intraoperatively recorded from the lesion using a depth electrode, ${ }^{5}$ suggesting that intralesional activity may have spread to the brainstem nuclei and resulted in seizures. A similar pathophysiology by way of pathological medullocerebellar connections might have been involved in our patient. However, we performed neither ictal SPECT nor intraoperative depth recording. In LDD, FDG-PET often identifies hypermetabolism localized in the cerebellar lesion, as with our patient. ${ }^{8}$ This might suggest that tumor pathophysiology rather than functional hyperactivation is associated with frequent VAs. ${ }^{8}$ We thus could not clarify the contributions of neuronal network-mediated mechanisms on the pathophysiology of VAs from this case.

The pathophysiology of VAs in LDD remains unclear. However, the fact that subtotal resection of the cerebellar lesion attached to the dorsolateral medulla oblongata resulted in immediate suppression of VAs in our patient suggests that the pathophysiology of LDD can include tumor-like SOL effects on the vomiting center of the medulla oblongata, and possibly neuronal network-mediated mechanisms.

\section{Acknowledgments}

We are grateful to the patient and his parents for their understanding and consent to this publication.

\section{References}

1. Abel TW, Baker SJ, Fraser MM, Tihan T, Nelson JS, Yachnis
AT, et al: Lhermitte-Duclos disease: a report of 31 cases with immunohistochemical analysis of the PTEN/AKT/mTOR pathway. J Neuropathol Exp Neurol 64:341-349, 2005

2. Babic T, Browning KN: The role of vagal neurocircuits in the regulation of nausea and vomiting. Eur J Pharmacol 722:38-47, 2012

3. Baker PC, Bernat JL: The neuroanatomy of vomiting in man: association of projectile vomiting with a solitary metastasis in the lateral tegmentum of the pons and the middle cerebellar peduncle. J Neurol Neurosurg Psychiatry 48:11651168,1985

4. Becker DE: Nausea, vomiting, and hiccups: a review of mechanisms and treatment. Anesth Prog 57:150-157, 2010

5. Delande O, Rodriguez D, Chiron C, Fohlen M: Successful surgical relief of seizures associated with hamartoma of the floor of the fourth ventricle in children: report of two cases. Neurosurgery 49:726-731, 2001

6. Fredericks C: Disorders of the cerebellum and its connections, in Fredericks CM, Saladin LK (eds): Pathophysiology of the Motor Systems: Principles and Clinical Presentations. Philadelphia: Davis, 1996, pp 445-465

7. Hanai S, Okazaki K, Fujikawa Y, Nakagawa E, Sugai K, Sasaki M, et al: Hemifacial seizures due to ganglioglioma of cerebellum. Brain Dev 32:499-501, 2010

8. Klisch J, Juengling F, Spreer J, Koch D, Thiel T, Büchert M, et al: Lhermitte-Duclos disease: assessment with MR imaging, positron emission tomography, single-photon emission CT, and MR spectroscopy. AJNR Am J Neuroradiol 22:824-830, 2001

9. Nowak DA, Trost HA: Lhermitte-Duclos disease (dysplastic cerebellar gangliocytoma): a malformation, hamartoma or neoplasm? Acta Neurol Scand 105:137-145, 2002

10. Pleuvry BJ: Physiology and pharmacology of nausea and vomiting. Anaesth Intensive Care Med 16:462-466, 2015

\section{Disclosures}

The authors report no conflict of interest concerning the materials or methods used in this study or the findings specified in this paper.

\section{Author Contributions \\ Conception and design: Ono, Honda. Acquisition of data: Ono, Honda. Analysis and interpretation of data: Ono, Honda, Baba, Ito. Drafting the article: Ono, Somagawa, Honda. Critically revis- ing the article: Baba, Hiu, Ushijima, Toda, Sato, Ito, Tsutsumi. Reviewed submitted version of manuscript: all authors. Approved the final version of the manuscript on behalf of all authors: Ono. Study supervision: Baba, Tsutsumi.}

\section{Correspondence}

Tomonori Ono, Epilepsy Center/Department of Neurosurgery, National Nagasaki Medical Center, 2-1001-1 Kubara, Omura, Nagasaki 856-8562, Japan. email: onotomon@gmail.com. 